\title{
INERTIAL EFFECTS ON TEARING INSTABILITY
}

\author{
F. E. M. SilveirA \\ Centro de Ciências Naturais e Humanas, Universidade Federal do ABC, Rua Santa Adélia, 166, Bairro Bangu, \\ CEP 09.210-170, Santo André, SP, Brazil \\ francisco.silveira@ufabc.edu.br
}

\begin{abstract}
.
In this work, we explore inertial effects, due to charged species in a resistive plasma, on the tearing instability. The standard theory of tearing modes assumes a long wavelength limit. At shorter wave lengths, inertial effects can become important and the current density flowing in the fluid can acquire a finite relaxation time. The introduction of such a correction into the problem leads to an extension of the standard dispersion relation. In the long wave length limit, we recover the standard scaling of the growth rate $\gamma$ with the plasma resistivity $\eta$, namely $\gamma \sim \eta^{3 / 5}$. However, in the short wavelength limit, we find that the scaling of $\gamma$ with the relevant plasma parameters changes significantly due to the influence of inertia. Notwithstanding, the dependence of $\gamma$ on the relaxation time of the current density is not determined. In order to achieve such a description, we propose to further rediscuss the problem in the framework of the boundary layer technique.
\end{abstract}

Keywords: resistive instabilities, Ohm's law, current relaxation.

\section{Introduction}

The tearing instability was originally discussed by Furth, Killeen, and Rosenbluth [1, and further developed by several authors 26 . It plays a central role in tokamak physics because disruptions of magnetized plasma columns (due to formation of magnetic islands) can occur and the confining chamber can become subjected to strong mechanical stresses [711. In astrophysical plasmas, tearing modes play an equally relevant role since a modification of magnetic topology (magnetic reconnection) can provoke conversion of magnetic energy into kinetic and thermal energies, and particle acceleration [12 17].

Tearing modes occur in an infinite plasma which is assumed to be ideal throughout the whole space, except in a thin current slab of width $a$, with a finite resistivity $\eta$. Then, by adopting Cartesian coordinates $(x, y, z)$, the equilibrium magnetic field, parallel to the $y z$-plane of the slab, exhibits a shear through the dependence of its $y$-component, $B_{y}$, on the $x$ coordinate, perpendicular to the constant equilibrium current density, $J_{0}$, which flows along the $z$-direction. Actually, all equilibrium fields, $F$, are assumed to depend only on the $x$-coordinate. Thus, a Fourier analysis can be carried-out on the assumption that the perturbative fields $f=f(x) \exp (\gamma t+\imath k y)$, with $|f| \ll|F|$. In the standard approach to the problem, the $x$-component of the perturbative magnetic field, $b_{x}$, satisfies the induction equation

$$
\gamma b_{x}=\imath \mu_{0} J_{0} k x v_{x}+\frac{b_{x}^{\prime \prime}}{\tau_{\mathrm{D}} / a^{2}},
$$

where $v_{x}$ denotes the $x$-component of the perturbative velocity field (which is assumed to be divergenceless), $\tau_{\mathrm{D}}=a^{2} \mu_{0} / \eta$ represents the magnetic diffusion time scale ( $\mu_{0}$ is the vacuum magnetic permeability), and the double prime stands for the second derivative of $b_{x}$ with respect to $x$.

\section{Ohm's law}

While deriving Eq. (1) in the standard approach, it is assumed that $\nabla^{2} b_{x} \approx b_{x}^{\prime \prime}$. However, this assumption clearly implies $\gamma \tau_{\mathrm{D}} \gg(k a)^{2}$. This means that as soon as $\gamma \tau_{\mathrm{D}} \sim(k a)^{2}$, such a long wave length limit cannot be justified. At shorter wave lengths, inertial effects due to charged species in the plasma can become important. In this case, the standard form of Ohm's law must be corrected by a time dependent term (a still more general formula should include ion and electron pressure gradients, as well as the Hall effect [18 21, however, those terms are not relevant for our purposes),

$$
\vec{E}+\vec{V} \times \vec{B}=\eta\left(1+\tau_{\mathrm{C}} \frac{\partial}{\partial t}\right) \vec{J}
$$

where $\vec{E}$ and $\vec{B}$ denote the electric and magnetic fields, respectively, and $\vec{V}$ and $\vec{J}$ represent the fluid flow and current density, respectively.

For singly ionized, approximately neutral, resistive plasmas, $\tau_{\mathrm{C}}=m_{\mathrm{e}} /\left(n_{\mathrm{e}} e^{2} \eta\right)$, where $m_{\mathrm{e}}, n_{\mathrm{e}}$, and $e$ denote the electron mass, number density, and charge, respectively. Actually, $\tau_{\mathrm{C}}$ can be interpreted as the finite relaxation time of the current density flowing in the plasma. Indeed, if $\vec{E}$ and $\vec{B}$ are suddenly removed from the presence of the fluid, then Eq. (2) shows that $\vec{J}(t)=\vec{J}(0) \exp \left(-t / \tau_{\mathrm{C}}\right)$, i. e., the initial current density $\vec{J}(0)$ damps off in the plasma, in a finite time interval of the order of $\tau_{\mathrm{C}}$. In the limit $\tau_{\mathrm{C}} \rightarrow 0$, the initial current $\vec{J}(0)$ is instantaneously 
damped, inertial effects are negligible, and sufficiently long wave lengths are enough to characterize the time dependence of the fields. In this case, Eq. 2 recovers the standard form of Ohm's law, $\vec{E}+\vec{V} \times \vec{B}=\eta \vec{J}$.

\section{Basic equations}

By introducing Eq. (2) in the problem, Eq. (1) is corrected to

$$
\left[\gamma+\frac{\left(1+\gamma \tau_{\mathrm{C}}\right)}{\tau_{\mathrm{D}} /(k a)^{2}}\right] b_{x}=\imath \mu_{0} J_{0} k x v_{x}+\frac{\left(1+\gamma \tau_{\mathrm{C}}\right) b_{x}^{\prime \prime}}{\tau_{\mathrm{D}} / a^{2}} .
$$

Linearization of the equation of motion shows that the $x$-component of the perturbative velocity field, $v_{x}$, satisfies the expression

$$
\gamma \rho_{0} v_{x}^{\prime \prime}=\imath J_{0} k x b_{x}^{\prime \prime}
$$

where $\rho_{0}$ denotes the (constant) mass density of the fluid which is assumed to be incompressible. Eqs. (3) and (4) are the basic equations for our approach.

\section{Growth rate}

The system of the two coupled differential equations (3) and (4) has to be solved both in the outer (ideal) and inner (resistive) plasma regions, and then the found solutions must be matched on the boundaries of the slab, at $|x|=a / 2$. In the linear theory of tearing modes, one shows that this condition is satisfied by requiring that the logarithmic jump

$$
\Delta^{\prime}=\frac{1}{b_{x}} \int_{0_{-}}^{0_{+}} b_{x}^{\prime \prime} d x
$$

of the $x$-component of the perturbative magnetic field, $b_{x}$, across $x=0$ (in the so-called resistive layer), be the same for both solutions (the so-called constant magnetic flux limit).

Except for much simplified equilibrium models (recall, for instance, the well-known Harris model [22]), Eq. (5) has to be treated numerically in the ideal plasma region [23-27]. However, $\Delta^{\prime}$ can be evaluated analytically in the resistive layer, with the help of a number of mathematical techniques. By following Goldston and Rutherford [28, one may easily check that the logarithmic jump satisfies the general dispersion relation

$$
\frac{\Delta^{\prime} a}{2.12}=\left[\frac{\gamma \tau_{\mathrm{D}}+\left(1+\gamma \tau_{\mathrm{C}}\right)(k a)^{2}}{\gamma \tau_{\mathrm{D}}\left(1+\gamma \tau_{\mathrm{C}}\right)^{3 / 4}}\right] \gamma^{5 / 4} \tau_{\mathrm{D}}^{3 / 4} \tau_{\mathrm{A}}^{1 / 2}
$$

in the resistive layer, where use has been made of Gamma functions 29] in order to compute the integral in Eq. (5). In Eq. (6), we have introduced the Alfvén time $\tau_{\mathrm{A}}=a / V_{\mathrm{A}}$, with $V_{\mathrm{A}}=\left(J_{0} / k\right) \sqrt{\mu_{0} / \rho_{0}}$ standing for the Alfvén speed. We see that if $\Delta^{\prime}>0$, then $\gamma>0$ too, and thus the latter can be interpreted as the growth rate of the unstable modes.

\section{Asymptotic scalings}

In the long wavelength limit, $\gamma \tau_{\mathrm{D}} \gg(k a)^{2}$, inertial effects, due to charged species in the plasma, can be neglected, $\gamma \tau_{\mathrm{C}} \ll 1$, and Eq. (6) approaches

$$
\frac{\Delta^{\prime} a}{2.12}=\gamma^{5 / 4} \tau_{\mathrm{D}}^{3 / 4} \tau_{\mathrm{A}}^{1 / 2}
$$

We see that Eq. (7) recovers the standard result of the linear theory of tearing modes, which establishes that the growth rate $\gamma$ scales with the plasma resistivity $\eta$ as $\gamma \sim \eta^{3 / 5}[1$.

However, in the opposite short wavelength limit, $\gamma \tau_{\mathrm{D}} \ll(k a)^{2}$, inertial effects can become important and Eq. (6) can be approximated to

$$
\frac{\Delta^{\prime} a}{2.12}=\left(1+\gamma \tau_{\mathrm{C}}\right)^{1 / 4}(k a)^{2} \gamma^{1 / 4} \tau_{\mathrm{D}}^{-1 / 4} \tau_{\mathrm{A}}^{1 / 2} .
$$

Interestingly, we see that the influence of inertia provokes a significant change on the scaling of the growth rate with the relevant plasma parameters. Notwithstanding, Eq. (8) does not determine the dependence of $\gamma$ on the inertial parameter $\tau_{\mathrm{C}}$. To circumvent this difficulty, we propose to further rediscuss the problem by making use of the so-called boundary layer technique 30. This technique can promote the rescaling of Eqs. (3) and (4) through the identification of a parameter which, although be irrelevant in the ideal region, can become relevant in the resistive layer. This parameter can be identified through the comparison of the order of magnitude of the various time scales involved. The proposed approach may provide the scaling of $\gamma$ with $\tau_{\mathrm{C}}$ when inertial effects become important, at sufficiently short wavelengths.

\section{Conclusion}

In this work, we have explored inertial effects, due to charged species in a resistive plasma, on the tearing instability. The standard theory of tearing modes assumes a long wavelength limit, as shown by Eq. (1). At shorter wave lengths, inertial effects can become important and Ohm's law must be corrected to Eq. (2). The introduction of such a correction into the problem has led to a general dispersion relation, as shown by Eq. (6).

In the long wave length limit, we have recovered the standard scaling of the growth rate $\gamma$ with the plasma resistivity $\eta$, namely $\gamma \sim \eta^{3 / 5}$. However, in the short wavelength limit, we have found that the scaling of $\gamma$ with the relevant plasma parameters changes significantly due to the influence of inertia. Nevertheless, the dependence of $\gamma$ on the inertial parameter $\tau_{\mathrm{C}}$ is not determined. In order to achieve such a description, we propose to further rediscuss the problem in the framework of the boundary layer technique.

\section{References}

[1] Killeen J. Rosenbluth M. N. Furth, H. P.

FiniteâĂ ̌resistivity instabilities of a sheet pinch. Phys. Fluids, 6:459, 1963. 
[2] Johnson J L Coppi B., Greene J M. The non-linear evolution of resistive interchange modes in a reversed-field pinch. Nucl. Fusion, 6:101, 1961.

[3] Johnson J L Glasser A. H., Greene J M. Resistive instabilities in general toroidal plasma configurations. Phys. Fluids, 18:875, 1975.

[4] Pellat R Rosenbluth M N Rutherford P H Sov. J. Coppi B., Galvão R M O. Sov. J. Plasma Phys., 2, 1976.

[5] Dependence of ideal-mhd kink and ballooning modes on plasma shape and profiles in tokamaks. Phys. Rev. Lett., 38:826, 1977.

[6] J. Mondt J. P., Weiland J. Nonlinear theory of large-mode-number ballooning modes in fully toroidal geometry. Plasma Phys., 34:143, 1985.

[7] Rosenbluth M N Waddell B V White R. W., Monticello D A. Saturation of the tearing mode. Phys. Fluids, 20:800, 1977.

[8] Wesson J Turner M. F. Nucl. Fusion, 22:1069, 1982.

[9] Sov. J. Smolyakov A. I. Plasma Phys., 15:667, 1989.

[10] Matsumoto H McGuire K Peebles W A Ritz Ch P Terry P W Zweben S J Wootton A. J., Carreras B A. Fluctuations and anomalous transport in tokamaks. Phys. Fluids B, 2:2879, 1990.

[11] Kadomtsev B. Plasma transport in tokamaks. Nucl. Fusion, 31:1301, 1991.

[12] Parker E. N. Sweet's mechanism for merging magnetic fields in conducting fluids. J. Geophys. Res., 62:509, 1957.

[13] Biskamp D. Magnetic reconnection via current sheets. Phys. Fluids, 29:1520, 1986.

[14] Sridhar S Goldreich P. Toward a theory of interstellar turbulence. 2: Strong alfvenic turbulence. Astrophys. J., 438:763, 1995.

[15] Vishniac E Lazarian A. Reconnection in a weakly stochastic field. Astrophys. J., 517:700, 1999.

[16] Vishniac E Otmianowska-Mazur K Kowal G., Lazarian A. Numerical tests of fast reconnection in weakly stochastic magnetic fields. Astrophys. J., 700:63, 2009.

[17] Vishniac E Otmianowska-Mazur K Kowal G., Lazarian A. Reconnection studies under different types of turbulence driving. Nonlinear Proc. Geoph., 19:297, 2012 .

[18] Spitzer L. Physics of Fully Ionized Gases. Second edition. Dover Publications, New York, 1969.

[19] Silveira F. E. M. Alfven waves and current relaxation: attenuation at high frequencies and large resistivity. J. Phys.: Conf. Ser., 370:012005, 2012.

[20] Silveira F. E. M. Magnetosonic waves and current relaxation. J. Plasma Phys. and Technol., 79:45, 2013

[21] Galvão R M O Silveira F. E. M. Magnetorotational instability, current relaxation, and current-vortex sheet. Phys. Plasmas, 20:082126, 2013.

[22] Harris E. G. On a plasma sheath separating regions of oppositely directed magnetic field. Nuovo Cimento, 23:115, 1962 .

[23] Bertotti B. Fine structure in current sheaths. Ann. Phys., 22:271, 1963.
[24] Haines M. G. Plasma containment in cusp-shaped magnetic fields. Nucl. Fusion, 17:811, 1977.

[25] Dawson J M Leboeuf J. N., Tajima T. Dynamic magnetic x points. Phys. Fluids, 25:784, 1982.

[26] Ion acceleration and direct ion heating in three-component magnetic reconnection. Phys. Rev. Lett., 76:3328, 1996.

[27] Kosugi T Somov B. V. Collisionless reconnection and high-energy particle acceleration in solar flares. Astrophys. J., 485:859, 1997.

[28] Rutherford P. H. Goldston R. J. Introduction to plasma physics. Institute of Physics, Bristol, 2000.

[29] Jeffreys B S Jeffreys H. Methods of Mathematical Physics. Third edition. Cambridge University Press, Cambridge, 1999.

[30] Orszag S A Bender C. M. Advanced Mathematical Methods for Scientists and Engineers: Asymptotic Methods and Perturbation Theory. Vol. 1. Springer-Verlag, New York, 1999. 